\title{
A study of rotating globular clusters
}

\section{The case of the old, metal-poor globular cluster NGC $4372^{\star, \star \star}$}

\author{
N. Kacharov ${ }^{1, \star \star \star}$, P. Bianchini ${ }^{2, \star \star \star}$, A. Koch ${ }^{1}$, M. J. Frank ${ }^{1}$, N. F. Martin ${ }^{3,2}$, G. van de Ven ${ }^{2}$, T. H. Puzia ${ }^{4}$, \\ I. McDonald ${ }^{5}$, C. I. Johnson ${ }^{6}$, and A. A. Zijlstra ${ }^{5}$ \\ ${ }^{1}$ Landessternwarte, Zentrum für Astronomie der Universität Heidelberg, Königstuhl 12, 69117 Heidelberg, Germany \\ e-mail: n.kacharov@lsw. uni-heidelberg.de \\ 2 Max Planck Institut für Astronomie, Königstuhl 17, 69117 Heidelberg, Germany \\ 3 Observatoire astronomique de Strasbourg, Université de Strasbourg, CNRS, UMR 7550, 11 rue de l'Université, 67000 Strasbourg, \\ France \\ ${ }^{4}$ Institute of Astrophysics, Pontifica Universidad Católica de Chile, Avenida Vicuña Mackenna 4860, Macul, Santiago, Chile \\ 5 Jodrell Bank Centre for Astrophysics, Alan Turing Building, Manchester M13 9PL, UK \\ ${ }^{6}$ Harvard-Smithsonian Center for Astrophysics, MS-15, 60 Garden Street, Cambridge MA 02138, USA
}

Received 25 February 2014 / Accepted 2 June 2014

\section{ABSTRACT}

\begin{abstract}
Context. NGC 4372 is a poorly studied old, very metal-poor globular cluster (GC) located in the inner Milky Way halo.
Aims. We present the first in-depth study of the kinematic properties and derive the structural parameters of NGC 4372 based on the fit of a Plummer profile and a rotating, physical model. We explore the link between internal rotation to different cluster properties and together with similar studies of more GCs, we put these in the context of globular cluster formation and evolution.

Methods. We present radial velocities for 131 cluster member stars measured from high-resolution FLAMES/GIRAFFE observations. Their membership to the GC is additionally confirmed from precise metallicity estimates. We build a velocity dispersion profile and a systemic rotation curve using this kinematic data set. Additionally, we obtain an elliptical number density profile of NGC 4372 based on optical images using a Markov chain Monte Carlo fitting algorithm. From this, we derive the cluster's half-light radius and ellipticity as $r_{\mathrm{h}}=3.44^{\prime} \pm 0.04^{\prime}$ and $\epsilon=0.08 \pm 0.01$. Finally, we give a physical interpretation of the observed morphological and kinematic properties of this GC by fitting an axisymmetric, differentially rotating, dynamical model.

Results. Our results show that NGC 4372 has an unusually high ratio of rotation amplitude to velocity dispersion $\left(1.2 \mathrm{vs} .4 .5 \mathrm{~km} \mathrm{~s}{ }^{-1}\right)$ for its metallicity. This puts it in line, however, with two other exceptional, very metal-poor GCs: M 15 and NGC 4590 . We also find a mild flattening of NGC 4372 in the direction of its rotation. Given its old age, this suggests that the flattening is indeed caused by the systemic rotation rather than tidal interactions with the Galaxy. Additionally, we estimate the dynamical mass of the GC $M_{\text {dyn }}=2.0 \pm 0.5 \times 10^{5} M_{\odot}$ based on the dynamical model, which constrains the mass-to-light ratio of NGC 4372 between 1.4 and $2.3 M_{\odot} / L_{\odot}$, representative of an old, purely stellar population.
\end{abstract}

Key words. globular clusters: general - globular clusters: individual: NGC 4372 - Galaxy: halo

\section{Introduction}

For a long time globular clusters (GCs) have been viewed as spherically symmetric, non-rotating stellar systems, successfully described to first order (see Trager et al. 1995; McLaughlin \& van der Marel 2005) by spherical, isotropic models (e.g. King 1966; Wilson 1975). The increasing abundance of observational data, however, has revealed noticeable deviations from this simple picture. Indeed, radial anisotropy (Ibata et al. 2013), significant degree of mass segregation (Da Costa 1982), signatures of core-collapse (Newell \& Oneil 1978; Djorgovski \& King 1984), velocity dispersion inflated by binaries (Bradford et al. 2011),

\footnotetext{
* Based on observations made with ESO telescopes at the La Silla Paranal Observatory under programmes ID 088.B-0492(A), 088.D0026(D), 164.O-0561, 71.D-02191B.

$\star \star$ The kinematic and photometric data are only available at the CDS via anonymous ftp to cdsarc.u-strasbg. fr (130.79.128.5) or via http://cdsarc.u-strasbg.fr/viz-bin/qcat?]/A+A/567/A69

$\star \star \star$ Member of the International Max Planck Research School for Astronomy and Cosmic Physics at the University of Heidelberg, IMPRS-HD, Germany.
}

and mild deviation from sphericity often associated with the presence of tidal tails (White \& Shawl 1987; Odenkirchen et al. 2001; Belokurov et al. 2006; Chen \& Chen 2010) have been observed in these stellar systems. Additionally, significant amounts of internal rotation (Lane et al. 2011; Bellazzini et al. 2012; Bianchini et al. 2013) have been observed in most Milky Way GCs. This has motivated the development of dynamical models, including the effects of external tides, mass segregation, corecollapse, and binary stars (Gunn \& Griffin 1979; Küpper et al. 2010; Zocchi et al. 2012) as well as a significant degree of rotation (Wilson 1975; Satoh 1980; Davoust 1986; van de Ven et al. 2006; Fiestas et al. 2006; Varri \& Bertin 2012).

NGC 4372 is a relatively nearby $\left(R_{\odot}=5.8 \mathrm{kpc}\right)$ and yet neglected GC in the inner halo. Photometric studies have established it as an archetypical old (>12 Gyr) and metal-poor object, $[\mathrm{Fe} / \mathrm{H}] \simeq-2.1$ dex (Alcaino et al. 1991; Geisler et al. 1995; Rutledge et al. 1997; Rosenberg et al. 2000; Piotto et al. 2002). It is also of particular interest from a dynamical point of view, since it is known to harbour close stellar binaries and luminous X-ray sources (Servillat et al. 2008). Curiously, these are mainly found outside the central regions. This is in contrast 
to the expected segregation based on the larger dynamical mass of such binary systems and suggests that NGC 4372 has still not established a significant degree of kinetic energy equipartition or that other dynamical processes have stirred up the cluster and expelled these sources from the centre. With a core relaxation time $\log t_{\mathrm{c}}=8.88 \mathrm{dex}$ (Harris 1996), NGC 4372 is an intermediately relaxed system, according to the classification of Zocchi et al. (2012). Considering its relatively low concentration and old age, it might be an example of a re-bounced, post-core-collapse GC (see Cohn \& Hut 1984). In this context, we also note its short orbital period $(0.1 \mathrm{Gyr})$ and moderate vertical space velocity component $\left(W=+100 \mathrm{~km} \mathrm{~s}^{-1}\right.$ ), so that the resulting orbit (Casetti-Dinescu et al. 2007) implies many slow disk crossings. The similarities of the orbit of NGC 4372 to the orbit of the massive GC NGC 2808 has suggested that both clusters are dynamically paired.

Internal rotation is one of the main reasons for the flattening of GCs, but external tides and pressure anisotropy can also play a significant role (van den Bergh 2008). In this work we build a dynamical, rotating model of the old, metal-poor, halo GC NGC 4372 using the Varri \& Bertin (2012) family of models (see also Bianchini et al. 2013). From this, we derive and use the maximum rotation amplitude to central velocity dispersion ratio $\left(A_{\text {rot }} / \sigma_{0}\right)$ to assess the importance of internal rotation in this GC. While it has been shown by means of $N$-body simulations that internal rotation and tidal interactions significantly accelerate the dynamical evolution of GCs (Boily 2000; Ernst et al. 2007; Kim et al. 2008), it is not yet clear how they affect the formation and the earliest stages of GC evolution. In an attempt to shed some light on this question, we investigate the impact of internal rotation on various cluster parameters like horizontal branch morphology, age, metallicity, and chemical variations.

This paper is organised as follows. Section 2 describes the observations and the data reduction. Section 3 is dedicated to the derivation of the structural parameters of NGC 4372 from photometry. Section 4 is dedicated to a detailed kinematic study of this object. Our discussion is developed in Sect. 5, where we present the rotating model and compare the kinematic results of NGC 4372 with other Milky Way GCs with existing kinematic data.

\section{Observations and data reduction}

\subsection{Spectroscopy}

The targets were selected from archival FORS2 pre-imaging in the $B$ and $V$ filters (ESO-programme 71.D-02191B, P.I.: L. Rizzi) and the 2MASS catalogue (Cutri et al. 2003) to cover the entire span of NGC 4372's red giant branch (RGB) and includes a number of asymptotic giant branch (AGB) stars (Fig. 1). The spectroscopic observations were carried out in service mode on the nights of Feb. 11, Mar. 08, and Mar. 10, 2012 using the Fibre Large Array Multi Element Spectrograph (FLAMES) mounted at the UT2 (Kueyen) of the Very Large Telescope (VLT) on Paranal (Pasquini et al. 2002). Five observing blocks (OB) were executed in total (exposure time 2775 s per OB) using two different Medusa plates. On each mask 133 fibres were fed to the GIRAFFE spectrograph (using the HR13 grating, which covers the wavelength range 6100-6400 $\AA$ with spectral resolution $R \sim 22000$ ) and eight fibres were fed to the UVES spectrograph. Nineteen of the GIRAFFE fibres were dedicated to the sky and 112 to the RGB/AGB targets. Both plate settings include different targets with a large overlap between them.

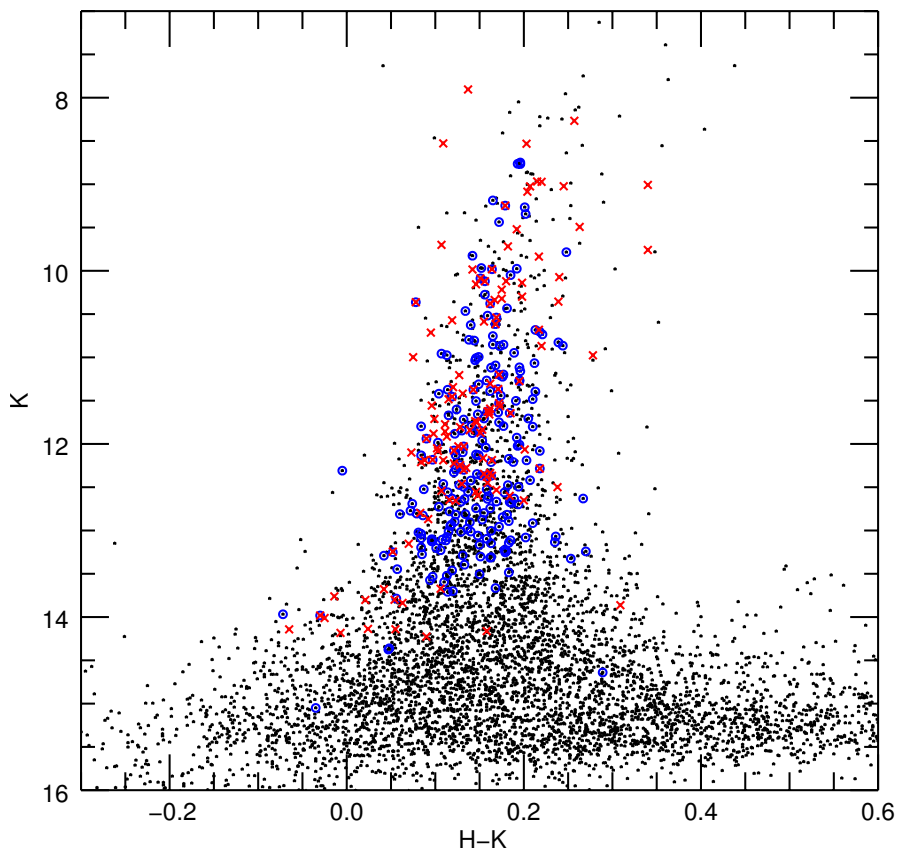

Fig. 1. 2MASS CMD of NGC 4372. The blue circles indicate all stars (both cluster members and foreground) in the first (088.B-0492) and red crosses indicate all stars in the second (088.D-0026) spectroscopic samples.

We reduced the FLAMES observations with the standard GIRAFFE pipeline, version 2.9.2 (Blecha et al. 2000). This pipeline provides bias subtraction, flat fielding, and accurate wavelength calibration from a Th-Ar lamp. The 19 sky spectra were combined and subtracted from the object spectra with the IRAF task skysub. We computed radial velocities of our targets by cross-correlating the spectra with a synthetic RGB spectrum with similar stellar parameters, as expected for our targets, using the IRAF fxcor task. All spectra were Doppler-shifted to the heliocentric rest frame and the individual spectra of the same stars (ranging from 2 to 5) were median combined using the IRAF scombine task. Finally, we normalised the spectra to the continuum level with the help of the IRAF continuum task. The final reduced, one-dimensional spectra have average signal-to-noise ratios (S/N) ranging from 20 to 200 per pixel, depending on the brightness of the stars and the number of the combined individual exposures. The data set consists of 108 different stars with successfully measured radial velocities, of which 64 were confirmed cluster members and the rest were identified as foreground stars.

In the following analysis, we also use a second GIRAFFE data set (ESO-programme 088.D-0026(D), P.I.: McDonald) of 123 stars observed in service mode on the nights of Jan. 15, Mar. 04, and Mar. 06, 2012 with the HR13 and HR14 GIRAFFE gratings, and thus, covering a total wavelength range from $6100 \AA$ to $6700 \AA$. We reduced this data-set with the girBLDRS ${ }^{1}$ software in a similar manner. The spectra predominantly cover the brighter RGB/AGB of NGC 4372 (Fig. 1) and are of high quality (median $\mathrm{S} / \mathrm{N}$ of $\sim 100$ per pixel). A radial velocity check and a metallicity estimate confirms 74 of the stars as cluster members, while the rest are classified as foreground contaminants. There is very little overlap between the two samples and we found only eight stars in common, five of which are cluster members. The

1 http://girbldrs.sourceforge.net/ 


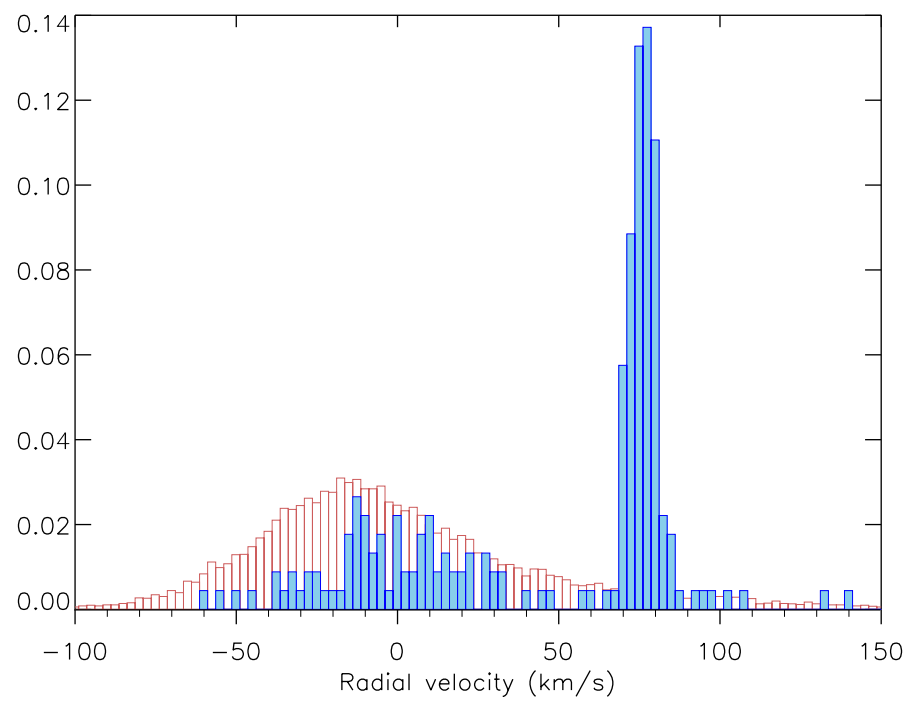

Fig. 2. Radial velocities' relative frequency of both spectroscopic samples (filled, blue boxes) overplotted over a Besançon model of the field stars in the direction of NGC 4372 (open, red boxes).

radial velocities of the common stars agree to within $1 \mathrm{~km} \mathrm{~s}^{-1}$ (mean difference $0.11 \mathrm{~km} \mathrm{~s}^{-1}$ with $1 \mathrm{~km} \mathrm{~s}^{-1}$ standard deviation).

For all stars in both data sets we also have metallicity estimates, which will be presented in a subsequent work. The membership of each cluster star is established based on simultaneous radial velocity and metallicity cuts.

Both data sets have essentially the same mean velocity and velocity dispersion, so we decided that it is safe to combine them. Our final NGC 4372 spectroscopic sample consists of 131 unique cluster member stars with $K$-band magnitude $\lesssim 15$ mag, confirmed from metallicity estimates $([\mathrm{Fe} / \mathrm{H}]<-2.0)$ of all the stars in the sample that have radial velocities between 50 and $100 \mathrm{~km} \mathrm{~s}^{-1}$. The median accuracy of the velocity measurements is $1 \mathrm{~km} \mathrm{~s}^{-1}$. In Fig. 2, we compare the radial velocities distribution of our full spectroscopic sample (cluster members plus foreground contamination) with the velocity distribution in this direction of the sky according to the Besançon model of the Galaxy (Robin et al. 2003). The Besançon model predicts about 2100 stars in an area of $0.2 \mathrm{deg}^{2}$ within our colour-magnitude selection limits. The mean radial velocity of NGC 4372 of $76 \mathrm{~km} \mathrm{~s}^{-1}$ is, however, quite distinct from the radial velocities of the majority of foreground stars, although a small contamination by such foreground stars is not excluded. But considering the very low metallicity of this $\mathrm{GC}([\mathrm{Fe} / \mathrm{H}] \simeq-2.2 \mathrm{dex})$ and the clearly metal-rich foreground stellar population, we are confident that we have selected a clean sample of NGC 4372 member stars. About 100 stars from the adopted Galactic model have radial velocities between 50 and $100 \mathrm{~km} \mathrm{~s}^{-1}$ and none of them is more metal poor than -1.8 dex. We come back to the detailed kinematics of these GC stars in Sect. 4.

\subsection{Photometry}

We used archival imaging obtained with the Wide Field Imager (WFI) at the $2.2 \mathrm{~m} \mathrm{MPG/ESO} \mathrm{telescope} \mathrm{at} \mathrm{La} \mathrm{Silla} \mathrm{(Baade} \mathrm{et} \mathrm{al.}$ 1999). We chose $V$ - and $I$-band observations of the cluster taken as part of the "pre-FLAMES" programme of the ESO Imaging Survey (programme 164.O-0561, PI: Krautter; cf. Momany et al. 2001). These observations cover a field of view (FOV) $30^{\prime} \times 30^{\prime}$ centred on the cluster. The basic data reduction, astrometric solution, and combining of mosaics were carried out using the
THELI pipeline (Erben et al. 2005; Schirmer 2013). We used the Two Micron All Sky Survey (2MASS) point source catalogue (Skrutskie et al. 2006) as astrometric reference and combined the observations to one stacked, undistorted image per filter. We then obtained instrumental magnitudes for unresolved objects using the DAOPHOT software package (Stetson 1987, 1993) from these images. We fixed photometric zero points to standard stars in the same field taken from the standard star database of Stetson $(2000,2005)^{2}$. Since we are primarily interested in relative photometry rather than absolute photometry in a given standard system, no colour term was included.

Since NGC 4372 resides behind a strip of Galactic gas and dust, it suffers from severe differential reddening (Hartwick \& Hesser 1973; Alcaino et al. 1991; Gerashchenko et al. 1999). In an attempt to correct for this effect, we followed the procedures described in Hendricks et al. (2012) and Milone et al. (2012). The main idea is to estimate the interstellar extinction for each star individually based on the median distance of its nearest neighbours to a fiducial line along the reddening vector. In our case, we used an adaptive number of the nearest neighbours depending on the density of the region, starting from 40 in the innermost $15^{\prime \prime}$ and using ten stars in the outermost regions. We used only main-sequence stars that have uncertainties on the $V$ - and $I$-band photometry less than $0.1 \mathrm{mag}$. A BASTI isochrone (Pietrinferni et al. 2004, 2006) of old age (15 Gyr) and low metallicity $(Z=0.0003)$, shifted to a distance modulus $(m-M)_{V}=15.0 \mathrm{mag}$ (Harris 1996), was used as a fiducial line, representative for this GC. Despite its relatively old age with respect to the age of the Universe, the chosen isochrone represents well the main sequence, the turn-off point, the RGB, and the magnitude of the horisontal branch (HB). We used the standard interstellar extinction law for the Milky Way where $A_{V}=3.1 \times E(B-V)=2.2 \times E(V-I)$ (Cardelli et al. 1989; Mathis 1990). The results of the de-reddening procedure are presented in Fig. 3, where we show a Hess diagram of the original raw colour-magnitude diagram (CMD) and the resulting CMD after applying the described algorithm. The de-reddening procedure also corrects for residual variations in the photometry that result from the variation of the point spread function or illumination (e.g. Koch et al. 2004) across the combined mosaics. We find a mean $E(B-V) \sim 0.5$ mag towards NGC 4372 in a good agreement with the Schlafly \& Finkbeiner (2011) re-calibration of the Schlegel et al. (1998) extinction map, with a significant variation between 0.3 and $0.8 \mathrm{mag}$ across the field of the GC. A reddening map is presented in Fig. 4.

To assess the completeness of the photometry, the final WFI photometric catalogue was compared to Hubble Space Telescope (HST) imaging of the central regions of the cluster (Piotto et al. 2002), which covers an area with a radius of about $1.5^{\prime}$ and can be safely assumed to be complete down to $V=21 \mathrm{mag}$. The HST F555W band magnitudes were calibrated to the WFI $V$-band extinction corrected magnitudes of matching targets. We assumed uniform extinction across the HST field, which is justified by its small size. We considered different magnitude ranges and counted the stars in both catalogues in six concentric rings each $15^{\prime \prime}$ thick assuming Poisson uncertainties. The results are presented in Fig. 5. Considering all of the stars, the comparison shows a completeness level of $(70 \pm 10) \%$ in the innermost $15^{\prime \prime}$ that rises to about $(85 \pm 5) \%$ at $90^{\prime \prime}$ from the cluster centre. This incompleteness is mostly due to the faintest stars in the sample with $V>20 \mathrm{mag}$, where the central $15^{\prime \prime}$ region has

\footnotetext{
2 Available at http://www3. cadc-ccda.hia-iha.nrc-cnrc.gc. $\mathrm{ca} /$ community/STETSON/standards/
} 

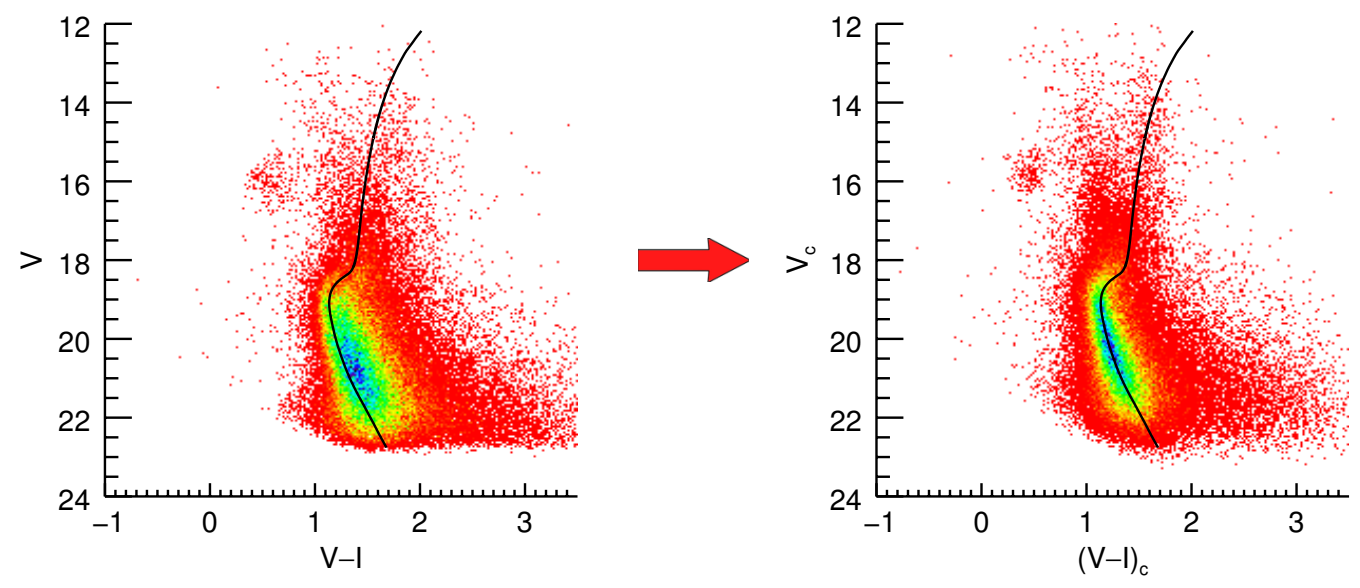

Fig. 3. Hess diagrams of NGC 4372's CMD before (left panel) and after (right panel) the differential reddening correction. The BASTI isochrone used as a fiducial line is superimposed.

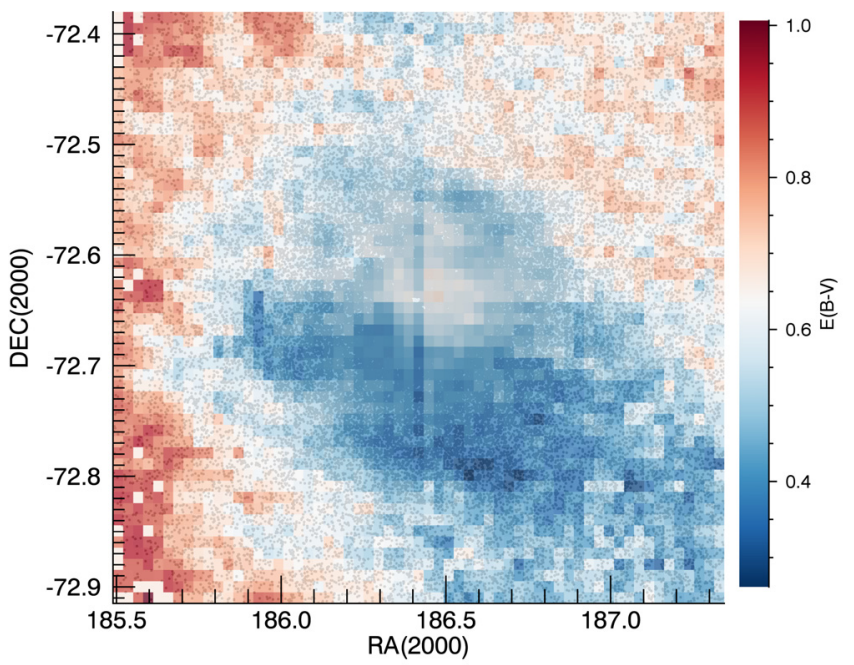

Fig. 4. A reddening map across the field of NGC 4372.

a completeness level of $(60 \pm 15) \%$ that rises to $(80 \pm 10) \%$ in the outer radii. The stars brighter than $V=20$ mag have a completeness level of about $(90 \pm 15) \%$ across the whole field. An exception is the region at $\sim 30^{\prime \prime}$, where we observe a sudden drop of completeness in the whole magnitude range.

\section{Structural parameters}

According to the Harris catalogue of GCs (Harris 1996, 2010 version $\left.^{3}\right)$, NGC 4372 has a half-light radius $r_{\mathrm{h}}=3.91^{\prime}$, a tidal radius $r_{\mathrm{t}}=34.9^{\prime}$, and a concentration parameter $c=\log \left(r_{\mathrm{h}} / r_{\mathrm{c}}\right)=$ 1.3 , where $r_{\mathrm{c}}=1.75^{\prime}$ is its core radius. These estimates come from a poorly constrained surface brightness profile presented in Trager et al. (1995). Furthermore, there is discrepant information for the ellipticity of NGC 4372 in the literature (White \& Shawl 1987; Chen \& Chen 2010). Therefore, we decided to rederive these parameters independently. It is interesting to check the direction of the flattening to assess the importance of internal rotation (see Sect. 4.2), or to give some insight on its tidal interaction with the Galaxy.

Using the WFI photometric catalogue, corrected for differential reddening and incompleteness in the central regions, we construct a number density, instead of a surface brightness profile, since the former is less sensitive to the presence of individual bright stars and foreground contaminants. It also allows us

\footnotetext{
3 http://physwww. mcmaster.ca/ harris/mwgc. dat
}

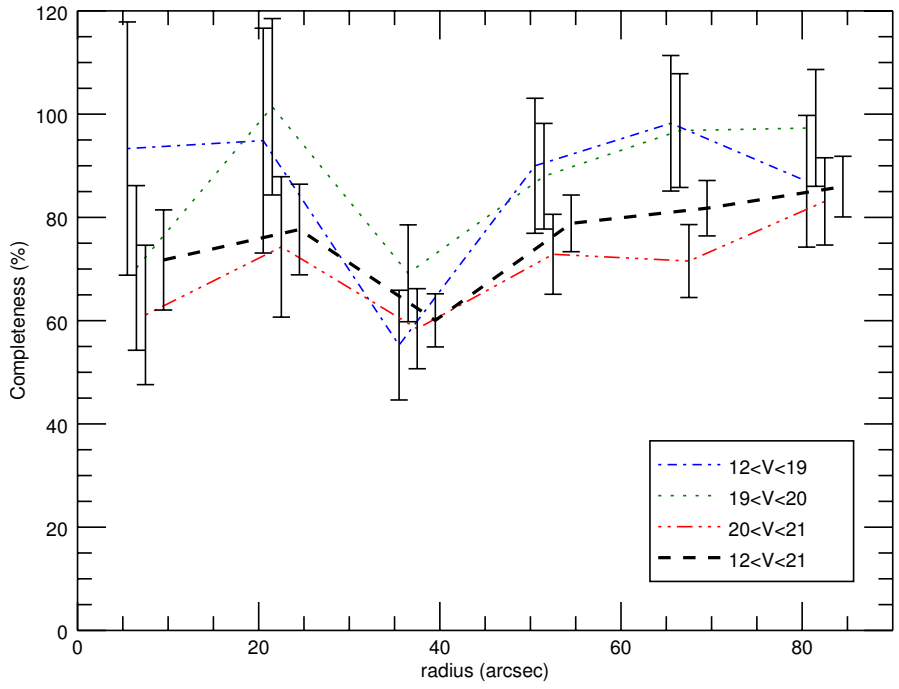

Fig. 5. Completeness of the WFI photometry compared to Hubble imaging of the innermost 1.5' of NGC 4372 in different magnitudes and spatial bins. The radial bins are shuffled by $1^{\prime \prime}$ for clarity.

to work with the full catalogue, instead with a limited number of surface bins. We only considered stars brighter than $V=20 \mathrm{mag}$, where we can safely assume that the sample is $100 \%$ complete beyond $90^{\prime \prime}$.

\subsection{The method}

We followed a modified version of the maximum likelihood method outlined in Martin et al. (2008) to fit the GC star number density profile. This approach consists of maximizing the loglikelihood function (Eq. (1)) by iterating a set of free parameters, for which the observations become most probable:

$\log \mathcal{L}\left(p_{1}, p_{2}, \ldots, p_{j}\right)=\sum_{i} \log \ell_{i}\left(p_{1}, p_{2}, \ldots, p_{j}\right)$

where $\ell_{i}\left(p_{1}, p_{2}, \ldots, p_{j}\right)$ is the probability of finding the measurement $i$ given the set of parameters $p_{1}, p_{2}, \ldots, p_{j}$. We used a set of six free parameters for our fit: the centroid of the cluster $\left(x_{0}, y_{0}\right)$, a model-dependent characteristic radius, the global ellipticity ${ }^{4}$ $(\epsilon)$, the orientation angle from north to east $(\theta)$ of the ellipse major axis, and the contaminating foreground density $\left(n_{\mathrm{f}}\right)$ in stars

\footnotetext{
4 The ellipticity is defined as $\epsilon=1-\frac{b}{a}$, where $a$ and $b$ correspond to the semi-major and semi-minor axes of the ellipse, respectively.
} 
per square arcmin. The centroid is defined as in van de Ven et al. (2006):

$x_{i}-x_{0}=\sin \left(\alpha_{i}-\alpha_{0}\right) \cos \delta_{i}$

$y_{i}-y_{0}=\sin \delta_{i} \cos \delta_{0}-\cos \delta_{i} \sin \delta_{0} \cos \left(\alpha_{i}-\alpha_{0}\right)$

where $\alpha_{i}$ and $\delta_{i}$ are the equatorial coordinates of the stars from the WFI catalogue and $\alpha_{0}=12^{\mathrm{h}} 25^{\mathrm{m}} 45.40^{\mathrm{s}}$ and $\delta_{0}=$ $-72^{\circ} 39^{\prime} 32.4^{\prime \prime}$ are the central cluster coordinates listed in the Harris catalogue. We chose to work with the projected Plummer family of models (Plummer 1911) of the following type:

$n(r)=n_{0}\left(1+\frac{r^{2}}{a^{2}}\right)^{-2}+n_{\mathrm{f}}$

In the Plummer profile, the characteristic radius $a$ corresponds to the half-light radius ${ }^{5}$ of the cluster. In the above equation, the independent variable $r$ is an elliptical radius (the semi-major axis of the adopted ellipse), which is related to the spatial position $(x, y)$ of the stars in the following way:

$r=\left\{\left[\frac{1}{1-\epsilon}(x \cos \theta-y \sin \theta)\right]^{2}+(x \sin \theta+y \cos \theta)^{2}\right\}^{1 / 2}$

and $n_{0}$ is the central number density of the cluster. The central number density is not a free parameter but constrained from the total number of stars in the FOV within the selection criteria $\left(N_{\text {tot }}\right)$. The equation

$N_{\mathrm{tot}}=\int_{\mathrm{FOV}} n(r) \mathrm{d} x \mathrm{~d} y=n_{0} \int_{\mathrm{FOV}}\left(1+\frac{r^{2}}{a^{2}}\right)^{-2} \mathrm{~d} x \mathrm{~d} y+A n_{\mathrm{f}}$

where $A$ is the total area of the FOV. From the last expression we can write that

$n_{0}=\frac{N_{\mathrm{tot}}-A n_{\mathrm{f}}}{\int_{\mathrm{FOV}}\left(1+\frac{r^{2}}{a^{2}}\right)^{-2} \mathrm{~d} x \mathrm{~d} y}$.

The integration from the above equation is done numerically over the entire FOV by dividing it into small segments with sizes $\Delta x, \Delta y$ much smaller than the expected half-light radius of the cluster (few arcmin).

We iterated the parameters in a Markov chain Monte Carlo (MCMC) manner following the Metropolis-Hastings algorithm (Hastings 1970), where each new set of parameters is derived randomly from the previous set, from a Gaussian distribution function with a defined standard deviation. The standard deviation for each parameter is chosen such as to optimise the acceptance rate of the Markov chain. A probability based on the likelihood is assigned to the new set of parameters and the chain is continued until we achieve a good sampling of the parameter space. The code was extensively tested with Monte Carlo drawn Plummer clusters with various half-light radii, ellipticities, and uniform background densities to confirm the correctness of the results. Muñoz et al. (2012) explored the conditions under which the outlined approach gives reliable results, namely the size of the FOV, the total number of stars, and the $n_{0} / n_{\mathrm{f}}$ ratio, which in the case of NGC 4372 are all fulfilled.

\footnotetext{
5 Technically, this is the radius that contains half of the stars. In order to relate this value to the half-light radius, one has to assume an initial mass function, binarity fraction, and segregation distribution. In this work, we use this quantity as a reasonable approximation of the half-light radius.
}

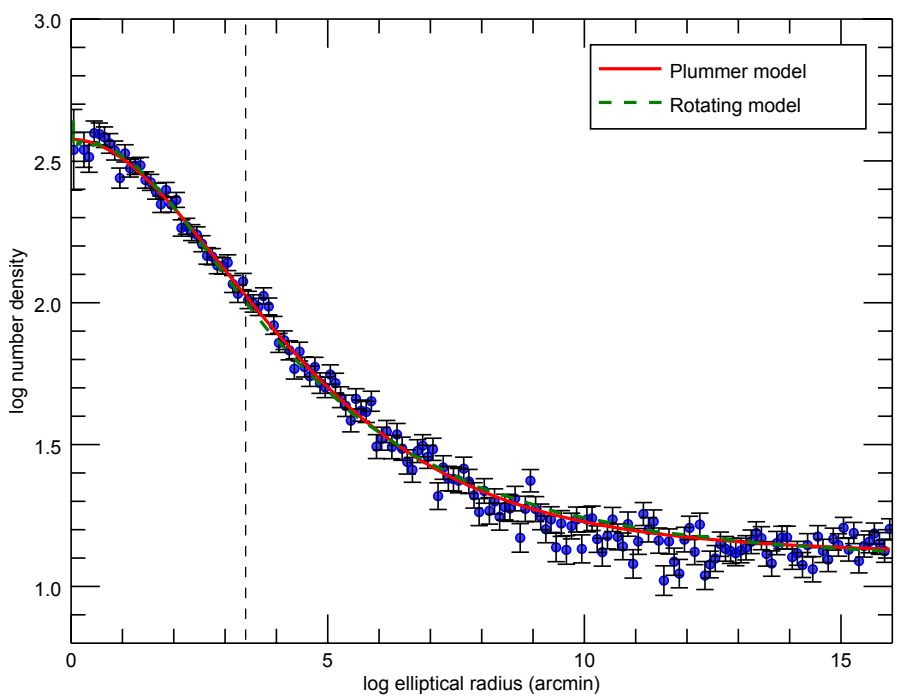

Fig. 6. Incompleteness corrected number density profile of NGC 4372 built in elliptical annuli of size $0.1^{\prime}$ (blue symbols) using stars brighter than $V=20$ mag. The best fitting Plummer model (red line), as well as the rotating model obtained (green line, see Sect. 5.1) are superimposed. The fitted half-light radius is indicated with a vertical, dashed line.

Table 1. Plummer model projected structural parameters from the MCMC fit.

\begin{tabular}{ccc}
\hline \hline & $V<20 \mathrm{mag}$ & $V<19 \mathrm{mag}$ \\
\hline$r_{\mathrm{h}}(\operatorname{arcmin})$ & $3.44 \pm 0.04$ & $3.03 \pm 0.06$ \\
$x_{0}(\operatorname{arcmin})$ & $-0.33 \pm 0.03$ & $-0.33 \pm 0.05$ \\
$y_{0}(\operatorname{arcmin})$ & $-0.64 \pm 0.03$ & $-0.62 \pm 0.04$ \\
$\epsilon$ & $0.08 \pm 0.01$ & $0.09 \pm 0.02$ \\
$\theta$ & $48^{\circ} \pm 6^{\circ}$ & $52^{\circ} \pm 6^{\circ}$ \\
$N_{0}{ }^{\circ}$ & $12000 \pm 100$ & $4200 \pm 70$ \\
$n_{\mathrm{f}}\left(\star / \square^{\prime}\right)$ & $12.82 \pm 0.16$ & $7.34 \pm 0.11$ \\
\hline
\end{tabular}

Notes. ${ }^{(1)} N_{0}=N_{\text {tot }}-A n_{\mathrm{f}}$ is the estimated number of stars belonging to the cluster, given our magnitude cuts.

\subsection{Profile fitting}

In order to obtain the global structural parameters of NGC 4372, we performed two fits, using all stars brighter than $V=20$ mag and $V=19 \mathrm{mag}$. In both cases, we assumed that the photometry is complete at radial distances greater than $1.5^{\prime}$, but we applied completeness corrections according to Fig. 5 in the central regions.

The results from the MCMC fits are summarised in Table 1. The uncertainty intervals in Table 1 are defined as the $1 \sigma$ deviation from the mean of a Gaussian representing the distribution function of each parameter from the Markov chain after excluding the "burn-in" iterations.

Figures 6 and 7 present the number density profiles of NGC 4372 obtained by using all stars brighter than $V=20$ mag and $V=19$ mag, respectively, built in confocal elliptical annuli with $0.1^{\prime}$ size that have ellipticities and position angles in accordance with the results reported in Table 1 for both fits. The best fitting Plummer profiles and rotating models (see Sect. 5.1) are also shown. We note here that the two curves drawn in the figures are not fit to the binned profiles; rather they are a result of the MCMC discrete fitting procedure in the case of the Plummer profile and the simultaneous modelling of the kinematics and number-density profiles in the case of the dynamical, rotating model. 


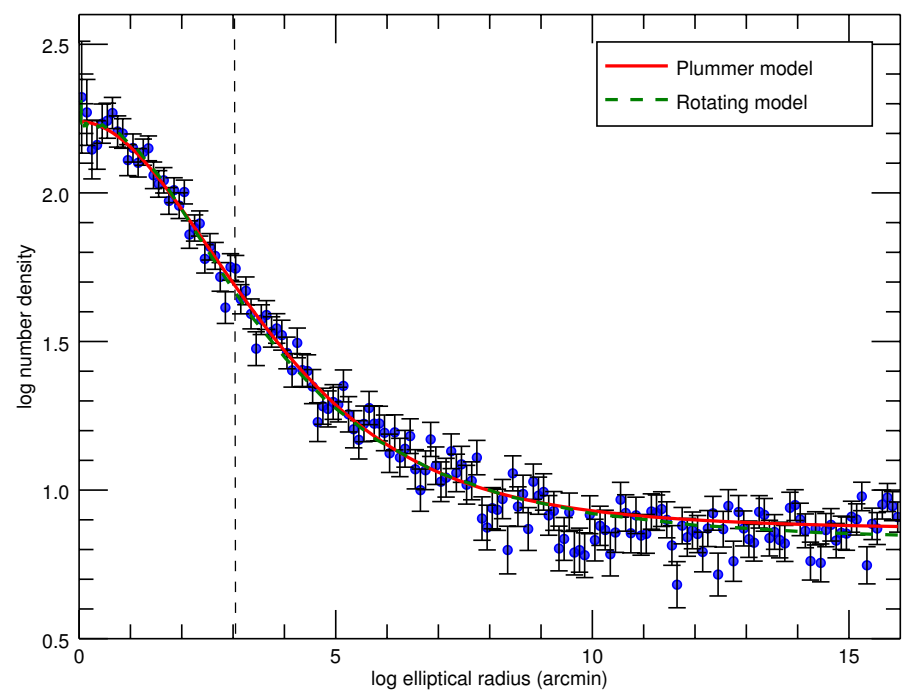

Fig. 7. Same as Fig. 6 but using only stars brighter than $V=19$ mag (the turn-off and RGB/AGB regions).

When using stars within different magnitude ranges the derived half-light radii vary significantly (see Table 1 ), while all other parameters used in the fit are consistent within the uncertainties. According to the BASTI isochrones, the stellar mass in the considered magnitude range varies between 0.70 and $0.75 M_{\odot}$. Because of this small contrast of masses, together with the overall uncertainties associated with the dereddening procedure and completeness estimation across the large WFI field, it seems difficult to draw conclusions about the mass segregation of the cluster. Instead, we attribute the more extended distribution profile of the fainter stars to still unaccounted incompleteness in the photometry. The very small HST field in which we have assesed incompleteness, however, does not allow us to properly account for incompleteness over the entire field of the WFI photometry. Another possible source of uncertainty comes from the dereddening procedure in which we are using a particular isochrone to correct for differential extinction the GC stellar population. This isochrone is not representative of the field population, which might be partly over-corrected, thereby increasing the relative fraction of contaminating field stars in the fainter samples, making number density fits artificially broader.

In the following analysis we apply the derived centroid corrections $\left(x_{0}, y_{0}\right)$ to the published coordinates of NGC 4372 in the Harris catalogue. The estimated coordinates of the centre are $\alpha_{0}=12^{\mathrm{h}} 25^{\mathrm{m}} 51^{\mathrm{s}}$ and $\delta_{0}=-72^{\circ} 38^{\prime} 57^{\prime \prime}$ for epoch 2000 .

\section{Kinematics}

As mentioned in Sect. 2.1, we measured radial velocities from individual exposures using the $f$ xcor task in IRAF. This method applies a Fourier cross-correlation between the spectra of interest and a given template and provides velocity error estimates based on the fitted peak height and the antisymmetric noise (Tonry \& Davis 1979). The adopted final velocities and their uncertainties are calculated as the error-weighted mean of the different velocity estimates for the same star from different exposures, ranging from 2 to 5 in our sample. For the second sample we averaged the radial velocities measured in the HR 13 and HR14 gratings for each star. The radial velocities derived from different exposures of the same star agree well with each other, showing that stellar binaries do not play a significant role.

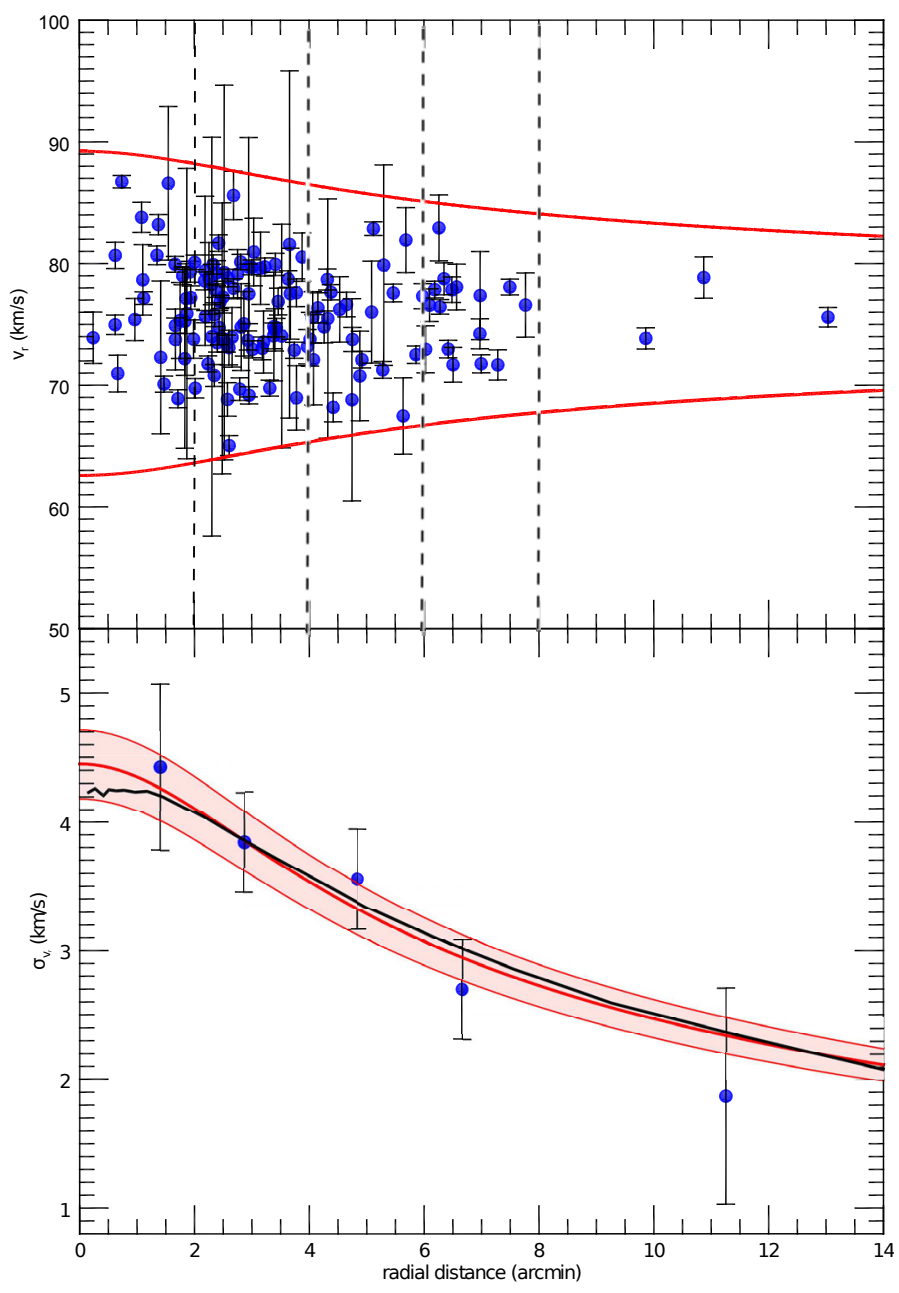

Fig. 8. Upper panel: radial velocities of the confirmed GC member stars versus radial distance from the centre of NGC 4372. The red curves indicate \pm 3 times the velocity dispersion as a function of the radial distance. The vertical dashed lines indicate the borders of the bins used to compute the velocity dispersion at a given radius. Bottom panel: velocity dispersion profile of NGC 4372 together with the best fitting Plummer profile (thick red line) and the rotating model (thick black line). The shaded area between the two thin red lines indicates the $1 \sigma$ uncertainty of the Plummer profile.

We used a maximum likelihood approach (Walker et al. 2006) to calculate the mean radial velocity of NGC $4372\left(v_{r}=\right.$ $\left.75.91 \pm 0.38 \mathrm{~km} \mathrm{~s}^{-1}\right)$ and its global intrinsic velocity dispersion $\left(\sigma=3.76 \pm 0.25 \mathrm{~km} \mathrm{~s}^{-1}\right)$ from the discrete velocity measurements. These values are in reasonable agreement with the radial velocity of $72.3 \pm 1.4 \mathrm{~km} \mathrm{~s}^{-1}$ and velocity dispersion of $4.3 \pm 0.9 \mathrm{~km} \mathrm{~s}^{-1}$, estimated by Geisler et al. (1995), based on 11 stars.

\subsection{Central velocity dispersion}

In order to estimate the central velocity dispersion $\sigma_{0}$ of NGC 4372, we divide the cluster into five radial bins and used the same maximum likelihood approach to estimate the velocity dispersion in each bin (Fig. 8), which we approximate with Plummer models. Plummer models describe isotropic stellar systems with constant density cores. We do not claim that this family of models is the best representation for this particular GC, rather we use it as a reasonable approximation. Our velocity dispersion does not allow us to distinguish between different types 
of models, such as the more physical King models for example. In principle, if mass follows light, as is expected to be the case for Galactic GCs (Lane et al. 2010), the characteristic radius $a$ should be equal to the half-light radius $r_{\mathrm{h}}$ of the cluster.

We fit the resulting velocity dispersion profile in a leastsquares sense with a projected Plummer (1911) model (Eq. (8)) using a characteristic Plummer radius $a=3.03^{\prime}$ estimated from the number-density profile for the brighter stars (RGB/AGB and turn-off stars; see Sect. 3.2) and setting the central velocity dispersion $\sigma_{0}$ as a free parameter:

$\sigma(r)^{2}=\frac{\sigma_{0}^{2}}{\sqrt{1+\frac{r^{2}}{a^{2}}}}$.

Although we have radial velocity measurements only for RGB/AGB stars in NGC 4372, using an estimate for the halflight radius value derived from the RGB/AGB and turn-off stars together is a compromise between having a statistically large sample of stars for constraining the number-density profile (see Fig. 7) and using stars with a similar spatial distribution.

Our data suggests a best-fit $\sigma_{0}=4.56 \pm 0.3 \mathrm{~km} \mathrm{~s}^{-1}$. The uncertainty is the formal $1 \sigma$ error computed from the co-variance matrix.

Having estimated the central velocity dispersion, it is straightforward to derive a dynamical mass for NGC 4372. According to an isotropic Plummer model the mass is obtained by the following expression (see e.g. Dejonghe 1987; Mackey et al. 2013):

$$
M=\frac{64 a \sigma_{0}^{2}}{3 \pi G} .
$$

Adopting a heliocentric distance of $5.8 \mathrm{kpc}$ (from the Harris catalogue), our radius translates to $a=5.1 \pm 0.05 \mathrm{pc}$ and we obtain $M=1.7 \pm 0.3 \times 10^{5} M_{\odot}$. This value is in agreement with the mass listed in Mandushev et al. (1991), $M=1.3_{(-0.8)}^{(+1.9)} \times 10^{5} M_{\odot}$ based on a mass-luminosity relation. The absolute magnitude of NGC 4372 is $M_{V}=-7.79 \mathrm{mag}$ according to the Harris catalogue (2010 version). This corresponds to a luminosity of $L_{V} / L_{\odot}=1.1 \times 10^{5}$ from which we can estimate the mass-tolight ratio for this GC to be $M / L_{V} \sim 1.5 M_{\odot} / L_{\odot}$, typical of most GCs (Mandushev et al. 1991; Pryor \& Meylan 1993). Thus, NGC 4372 is a typical representative of the old, purely stellar populations without detectable amounts of dark matter.

\subsection{Rotation}

We checked for systemic rotation in NGC 4372, following a well established method (see e.g. Mackey et al. 2013; Bellazzini et al. 2012; Lane et al. 2009; Lane et al. 2010, and references therein): to this end, we measured the difference between the maximum likelihood mean velocity on either side of a line passing through the cluster's centre and rotated at different position angles (Fig. 9). The resulting curve is well described by a sine law of the type $\Delta v_{r}=A_{0} \sin \left(\theta^{\prime}+\theta_{0}^{\prime}\right)$, where $\theta_{0}^{\prime}$ is the position angle of the rotation axis and $A_{0}$ corresponds to two times the amplitude of rotation modified by a factor of $\sin i$. The angle $i$ is the unknown inclination of the GC with respect to the line of sight. Since stellar proper motions for this object are rather unreliable, there is no way to estimate $\sin i$ (see for example Bianchini et al. 2013) and the amplitude of rotation is just a lower limit of the true intrinsic rotation velocity for NGC 4372. The results of the $\chi^{2}$-fit are $A_{0}=2.0 \pm 0.2 \mathrm{~km} \mathrm{~s}^{-1}\left(v_{\mathrm{rot}} \sin i=1.0 \pm 0.1 \mathrm{~km} \mathrm{~s}^{-1}\right)$ and

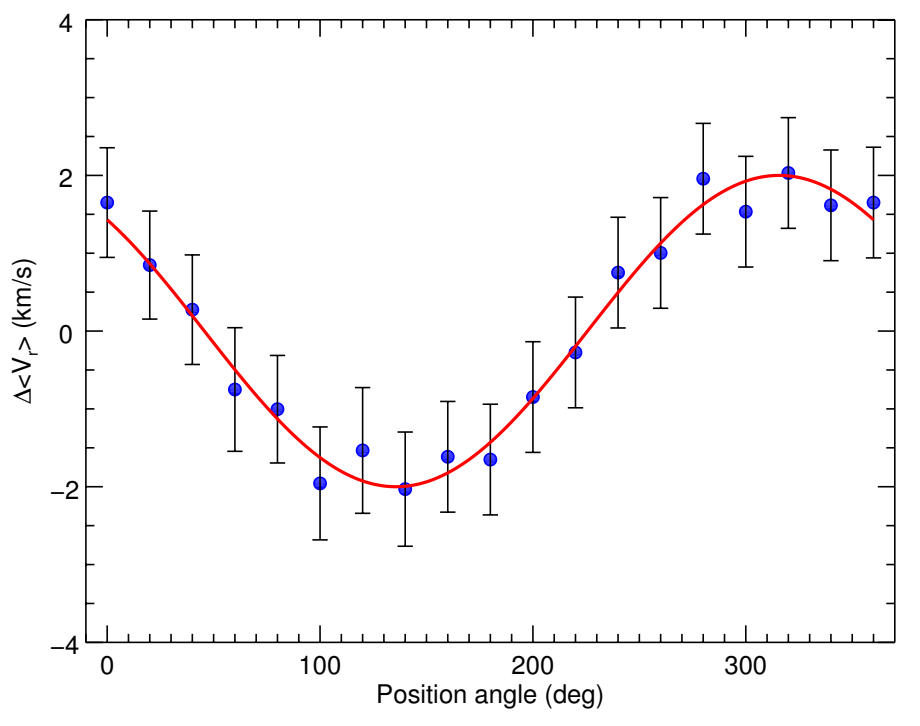

Fig. 9. Rotation in NGC 4372. The plot displays the difference between the mean velocities on each side of the cluster with respect to a line passing through its centre at a given position angle (measured from north to east, north $=0^{\circ}$, east $=90^{\circ}$ ). The red line is the sine function that best fits the data.

the projected rotation axis lies at a position angle of $136^{\circ} \pm 7^{\circ}$, measured from north through east. The results remain the same if we fit the sine law to the discrete velocities of the sample at the position angle of each star, rather than to the binned picture presented in Fig. 9.

The amplitude of rotation, however, varies with the radius as naturally expected with the presence of differential rotation (e.g. Bellazzini et al. 2012; Mackey et al. 2013). The simplest way to show this for NGC 4372 is to construct a rotation profile (Fig. 10). The figure shows the mean difference between the velocity measured in different overlapping bins along an axis perpendicular to the rotation axis and the systemic radial velocity of the cluster. The resulting curve was then fitted by a simple rotation profile of the form (as in Mackey et al. 2013):

$v_{\text {rot }}=\frac{2 A_{\text {rot }}}{r_{\text {peak }}} \times \frac{X_{\theta_{0}^{\prime}}}{1+\left(X_{\theta_{0}^{\prime}} / r_{\text {peak }}\right)^{2}}$

where $r_{\text {peak }}$ is the projected radius at which the maximum amplitude of rotation $A_{\text {rot }}$ is measured and $X_{\theta_{0}^{\prime}}$ is the distance in arcmin from the cluster's centre along an axis perpendicular to the axis of rotation. For NGC 4372, we found $A_{\text {rot }}=1.2 \pm 0.25 \mathrm{~km} \mathrm{~s}^{-1}$ at $r_{\text {peak }}=1.3 \pm 0.5^{\prime}$ from the cluster centre. The estimated $A_{\text {rot }} / \sigma_{0}$ ratio is $0.26 \pm 0.07$. Its meaning is further discussed in Sect. 5 .

The estimated angle of the major axis of the ellipsoid that best fits the number-density profile $\theta \sim 40^{\circ}$ is perpendicular to the estimated position angle of the rotation axis of NGC 4372 $\left(\theta^{\prime}=136^{\circ}\right)$. Since our MCMC fitting algorithm is most sensitive to the ellipticity in the inner parts of the cluster and the maximum of rotation is found well within the half-light radius of NGC 4372, we can already conclude that systemic rotation is likely the main driver of the flattening of NGC 4372. Figure 11 shows the spatial extent of the RGB/AGB and turn-off stars from our photometric catalogue as well as the stars for which we have radial velocity measurements. The figure illustrates the good alignment between the estimated rotation axis and the direction of flattening of NGC 4372. 


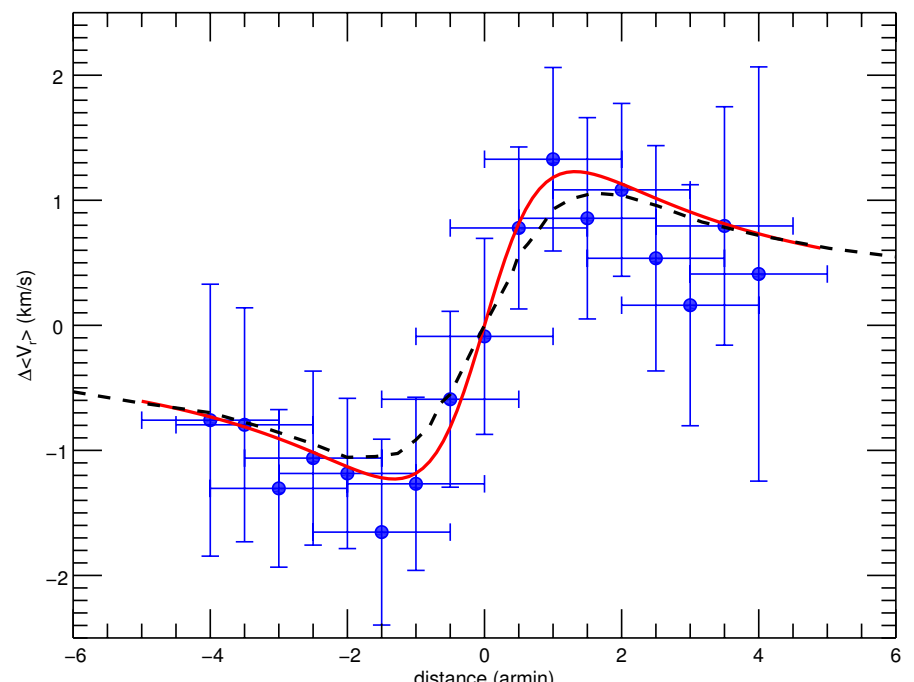

Fig. 10. Rotation profile of NGC 4372, where the abscissa shows the distance from the centre of the $\mathrm{GC}$ along the axis perpendicular to the rotation axis and the ordinate shows the mean offset from the GC's systemic velocity in different overlapping bins. The horizontal error bars indicate the size of the selected bins, while the vertical error bars indicate the formal uncertainty of the mean velocity offset. The best fit, according to Eq. (10), is overplotted with a red line and the rotating model is shown with a black, dashed line.

\section{Discussion}

\subsection{A dynamical model for NGC 4372}

The present section is motivated by the need to provide a global and realistic dynamical interpretation of NGC 4372, taking into consideration all the morphological and kinematic properties we have collected and discussed in the previous sections. In order to carry out a complete dynamical description of NGC 4372 we compare our full set of observations with a family of physically motivated models based on distribution functions, recently applied to a selected sample of GCs (Varri \& Bertin 2012; Bianchini et al. 2013). These self-consistent models have been specifically constructed to describe quasi-relaxed stellar systems and to take realistic differential rotation, axisymmetry, and pressure anisotropy into account. The models are defined by four dimensionless parameters (concentration parameter $\Psi$, rotation strength parameter $\chi$, and the parameters $\mu$ and $\xi$ determining the shape of the rotation profile). A full description of the distribution function and of the parameter space is provided in Varri \& Bertin (2012).

The comparison between the differentially rotating models and observations requires us to specify the four dimensionless parameters and five additional quantities: three physical scales (i.e. the radial scale $r_{0}$, the central surface density $n_{0}$, and the velocity scale $v_{0}$ ), the inclination angle $i$ between the rotation axis and the line-of-sight direction, and a foreground contamination term $n_{\mathrm{f}}$ (to be added to the surface density profile). For simplicity, we adopted two fixed inclination angles at $i=45^{\circ}$ and $i=90^{\circ}$. The fit procedure is conducted in two steps. First, we determine the dimensionless parameters such to reproduce the

\footnotetext{
6 The inclination angle of the rotating axis is generally not known for globular clusters; given the fact we observe small but yet significant flattening and rotation, we are led to conclude that the angle is likely to be different from $i=0$. Strictly statistically, $\langle\sin i\rangle=\frac{\pi}{4}$, therefore the average inclination angle is $\langle i\rangle=52^{\circ}$. A detailed exploration of this additional parameter is beyond the goal of our study.
}

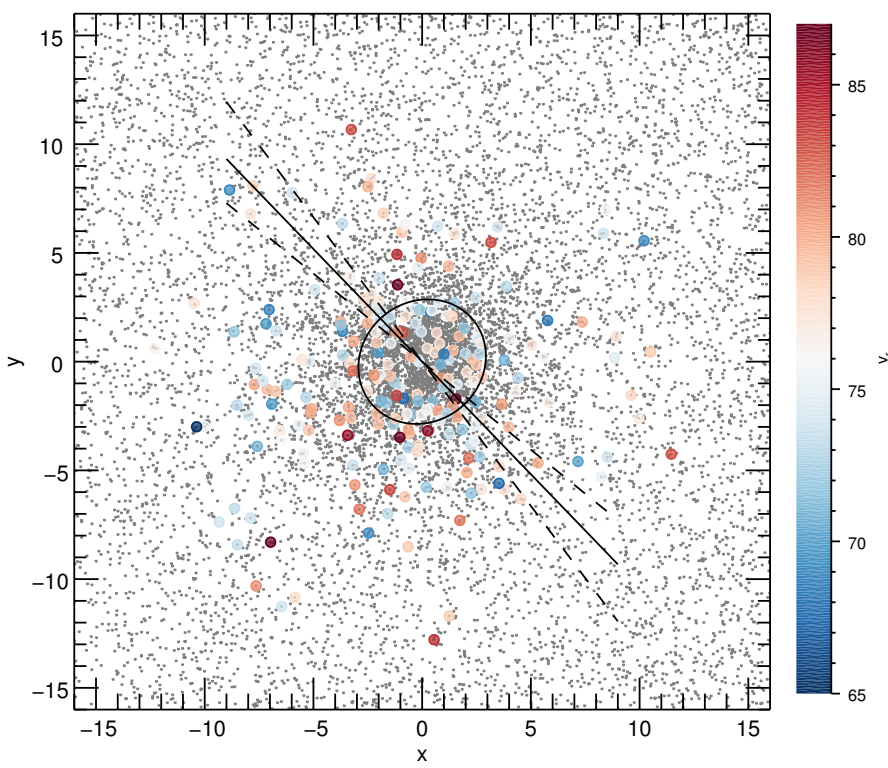

Fig. 11. Spatial extent of the RGB/AGB and turn-off stars of NGC 4372. Stars for which we have radial velocity measurements are highlighted with coloured symbols, denoting their line-of-sight velocity. The best fitting ellipse for this set of stars (see the last column of Table 1) and the rotation axis with its uncertainty are superimposed.

observed value of $A_{\text {rot }} / \sigma_{0}$ and the observed position of the rotation peak (for further details see Sects. 3.1 and 3.5 of Bianchini et al. 2013). Second, we calculate the physical scales by minimizing $\chi^{2}$ simultaneously for the combined photometry (surface density profile) and kinematics (dispersion profile and rotation profile). This provides at once all the constraints needed to determine the best-fit dynamical model.

In the subsequent dynamical analysis, stars will be used as kinematic tracers and we will assume that the stars in our kinematic data sets trace the true stellar mass population of the system. We recall that our dynamical models are one mass component models, and therefore can be applied when the stellar population of the system is homogeneous. In the case of the presence of mass segregation and energy equipartition, however, we expect stars of different masses to have different spatial distribution and different kinematics (Trenti \& van der Marel 2013).

Some additional attention is required when using simultaneously photometry and kinematics that refer to stars of different magnitude ranges. Therefore, we compute two models, where the first takes as an input the number density distribution of all stars brighter than $V=20$ mag (Fig. 6), and the second one uses only stars brighter than $V=19$ mag (Fig. 7). A better $\chi^{2}$ is obtained when only the number density profile of the brighter stars is used. The computed dimensionless parameters are as follows: $\Psi=5, \chi=0.16, \mu=0.5, \xi=3$. The derived physical scales and structural parameters (core radius, half-light radius, concentration, total mass, $M / L_{V}$ ) are reported in Table 2.

The best-fit model, constrained using the brighter RGB/AGB and turn-off stars, is able to reproduce satisfactorily both the photometric and the kinematic radial profiles. In particular, the model well reproduces the central region and the outer part of the number density profile (see Fig. 7). For the line-of-sight kinematic profiles, the model is able to reproduce simultaneously the shape of the velocity dispersion profile and of the rotation profile, matching the characteristic rigid rotation behaviour in the central regions, the velocity peak, and the subsequent decline (see Figs. 8 and 10). We ran a two-dimensional realization of the 
Table 2. Parameters derived from the rotating model.

\begin{tabular}{cccc}
\hline \hline & $V<20 \mathrm{mag}$ & $V<19 \mathrm{mag}$ & $V<19 \mathrm{mag}$ \\
& $i=45^{\circ}$ & $i=45^{\circ}$ & $i=90^{\circ}$ \\
\hline \multicolumn{4}{c}{ Best fit physical scales } \\
$r_{0}(\operatorname{arcmin})$ & $2.49 \pm 0.05$ & $2.19 \pm 0.06$ & $2.23 \pm 0.07$ \\
$v_{0}\left(\mathrm{~km} \mathrm{~s}^{-1}\right)$ & $4.81 \pm 0.25$ & $4.98 \pm 0.26$ & $5.14 \pm 0.28$ \\
$n_{0}$ & $358 \pm 8$ & $165.2 \pm 6.9$ & $168.2 \pm 10.8$ \\
$n_{\mathrm{f}}$ & $12.0 \pm 0.3$ & $6.7 \pm 0.23$ & $6.7 \pm 0.15$ \\
\hline \multicolumn{4}{c}{ Derived quantities } \\
$r_{\mathrm{h}}(\operatorname{arcmin})$ & $3.64 \pm 0.07$ & $3.20 \pm 0.09$ & $3.12 \pm 0.10$ \\
$r_{\mathrm{t}}(\operatorname{arcmin})$ & $37.3 \pm 0.75$ & $32.8 \pm 0.9$ & $33.4 \pm 1.0$ \\
$r_{\mathrm{c}}(\operatorname{arcmin})$ & $2.25 \pm 0.05$ & $1.98 \pm 0.05$ & $1.94 \pm 0.06$ \\
$c=\log \left(r_{\mathrm{t}} / r_{\mathrm{c}}\right)$ & $1.22 \pm 0.01$ & $1.23 \pm 0.02$ & $1.24 \pm 0.03$ \\
$M_{\mathrm{dyn}}\left(10^{5} M_{\odot}\right)$ & $1.88 \pm 0.38$ & $1.97 \pm 0.54$ & $1.94 \pm 0.61$ \\
$M / L_{V}\left(M_{\odot} / L_{\odot}\right)$ & $1.7 \pm 0.4$ & $1.8 \pm 0.5$ & $1.8 \pm 0.6$ \\
\hline
\end{tabular}

rotating model through the MCMC fitting routine to estimate its flattening due to rotation. Interestingly, the model does not allow significant deviations from spherical symmetry ${ }^{7}$.

The derived estimates of the total dynamical mass from both models of $M_{\text {dyn }}=1.9 \pm 0.4 \times 10^{5} M_{\odot}$ and $2.0 \pm 0.5 \times 10^{5} M_{\odot}$ are in agreement with the virial estimate reported in Section 4.1, and suggests a mass-to-light ratio $M / L_{V}$ between 1.4 and $2.3 M_{\odot} / L_{\odot}$.

To assess the impact of the unknown inclination angle, we also computed a model assuming the extreme case that we see the cluster edge on $\left(i=90^{\circ}\right)$. The derived parameters from this model are also reported in Table 2. Although this assumption provides slightly better $\chi^{2}$-fit, the derived structural quantities are essentially unchanged and we can conclude that a reasonable choice of the inclination angle (between $45^{\circ}$ and $90^{\circ}$ ) does not have a real impact in our final results. Inclination angles below $30^{\circ}$ make the rotation signal practically undetectable.

\subsection{On rotation and ellipticity}

While GCs are to a high degree spherically symmetric systems, mild deviations from the perfect sphere (ellipticities up to 0.20) are observed in most of them. Different reasons for what could cause the flattening are discussed in the literature. Amongst other reasons, internal rotation, pressure anisotropy, and external tides have been suggested to have a significant impact (see Goodwin 1997; Gnedin et al. 1999; van den Bergh 2008; Bianchini et al. 2013). Mackey \& van den Bergh (2005) also suggested that the observed shape of GCs could be governed by a tri-axial, dark matter, mini halo, in which GCs hypothetically could reside. Although the main paradigm is that there is little or no dark matter around GCs (Baumgardt et al. 2005, 2009; Lane et al. 2009; Lane et al. 2010; Sollima et al. 2012; Ibata et al. 2013), this subject is not yet fully examined because of the lack of radially extended kinematic data sets suitable for dynamical studies in the majority of GCs (Zocchi et al. 2012), unless tidal streams are observed (Mashchenko \& Sills 2005a,b).

Plots of the $A_{\text {rot }} / \sigma_{0}$ ratio vs. ellipticity (Fig. 12) are a common tool used to assess the importance of rotation in shaping stellar systems (Davies et al. 1983; Emsellem et al. 2011, in the context of elliptical galaxies). Both the rotational velocity estimates and the observed ellipticities depend on the unknown inclination angles to the line-of-sight and thus constitute lower limits. Taking that into account and the limited radial extent of

\footnotetext{
7 Complex interplay between rotation and velocity anisotropy can contribute to the final, morphological properties of the modelled stellar system.
}

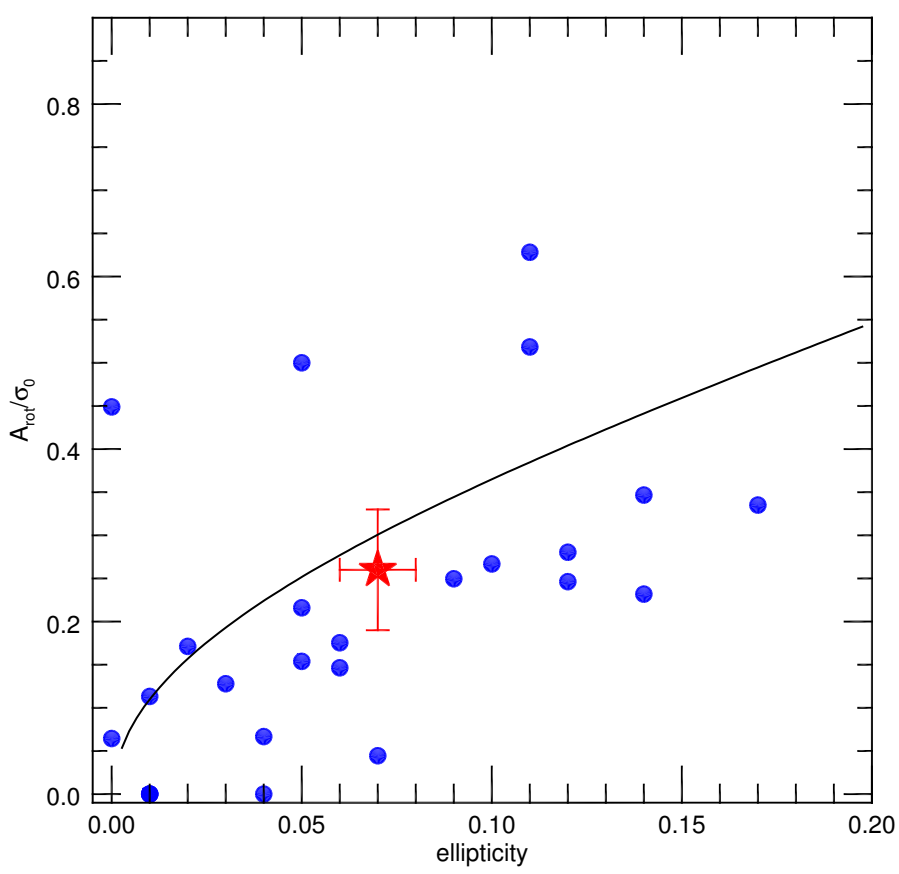

Fig. 12. Rotation-to-velocity dispersion ratio as a function of GC ellipticity. The sample of GCs with known $A_{\text {rot }} / \sigma_{0}$ values comes from Bellazzini et al. (2012); Bianchini et al. (2013) and the ellipticity information is taken from Harris (1996, 2010 version), except for NGC 4372 (red star), for which we use our own estimates. A model of an isotropic, rotating spheroid is superimposed for comparison (Binney 2005).

the available radial velocity data, most of the GCs plotted in Fig. 12 have ellipticities consistent with the flattening caused by rotation according to a model of a self-gravitating, rotating sphere (Binney 2005). Although the plot shows that in most cases the flattening indeed seems to be caused by significant internal rotation, this is difficult to be proven conclusively with the existing data sets. Both rotation and ellipticity vary with the radial distance from the cluster centre (Geyer et al. 1983; Bianchini et al. 2013), and different factors (including anisotropy) may have different impact at different projected radii. There is, for example, a large systematic discrepancy between White \& Shawl (1987) and Chen \& Chen (2010), the two most extensive studies on GCs' ellipticities. They are based on different types of data and methods, which are sensitive to different radial distances.

NGC 4372 lies firmly on the sequence described by other GCs in the $A_{\text {rot }} / \sigma_{0}$ vs. $\epsilon$ diagram (Fig. 12) and in good agreement with the dynamical model. This implies that its flattening is mostly caused by its significant internal rotation. This conclusion is additionally supported by the excellent alignment of the rotational axis and the orientation of the best fitting ellipse and is somewhat surprising given its proximity to the Galactic disk, where external tides are expected to play a significant role. The lack of proper motions prevents us from directly assessing the role of anisotropy in velocity space.

\subsection{How does rotation affect other GC parameters?}

Bellazzini et al. (2012) explored the dependence of various cluster parameters on the $A_{\text {rot }}$ and $A_{\text {rot }} / \sigma_{0}$ ratio using the kinematic results for a sample of 25 Galactic GCs. They found a very strong correlation between the amount of rotation in a GC and its horizontal branch (HB) morphology, namely that GCs with blue HBs are slower rotators than those with red or extended 

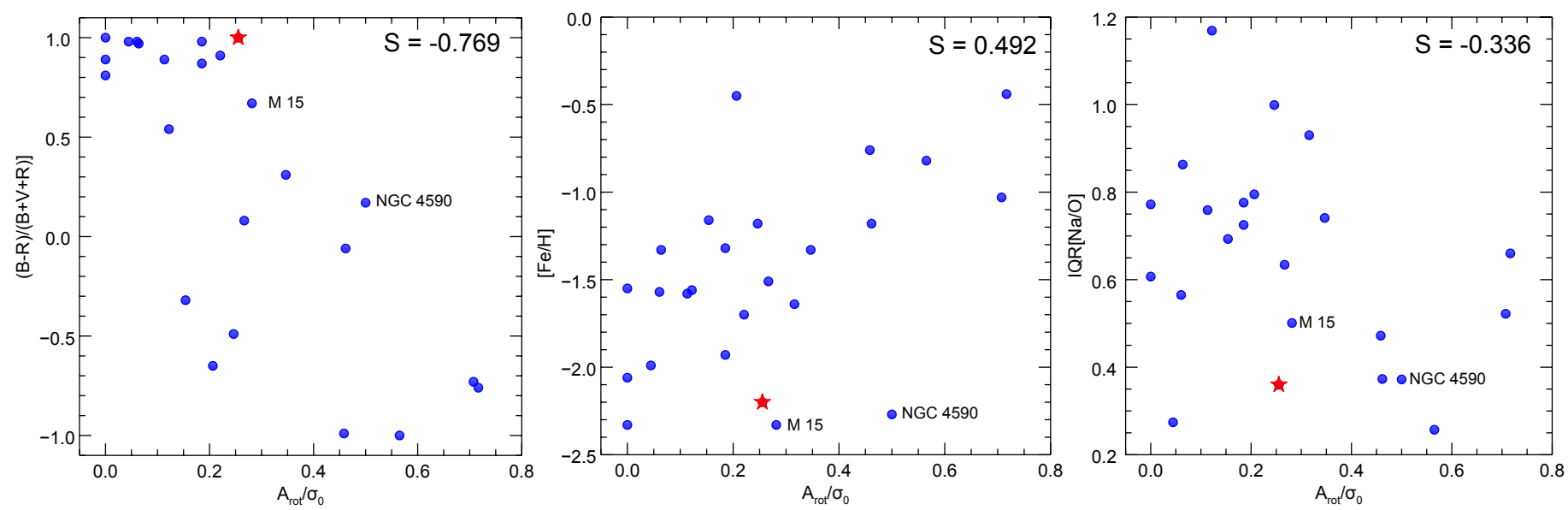

Fig. 13. $A_{\text {rot }} / \sigma_{0}$ ratio in a number of GCs as a function of different cluster parameters: horizontal branch morphology (left panel), [Fe/H] (mid panel), and IQR $[\mathrm{Na} / \mathrm{O}]$ (right panel). The Spearman rank correlation coefficient is shown in the upper right corner of each panel. Rotation data are taken from Bellazzini et al. (2012). NGC 4372 has a red star symbol.

HBs (Fig. 13). NGC 4372 is not an exception and firmly takes its place as a relatively slow rotator with a very blue $\mathrm{HB}$ on this diagram. The HB morphology, however, is one of the most complex parameters that characterise GCs (see Catelan 2009, for a detailed review) and although not completely unexpected, such a strong correlation of the rotation with the HB morphology is difficult to explain since it has to be due to the superposition of multiple effects. Metallicity is the first parameter that shapes the HB morphology (Lee et al. 1990; Fusi Pecci et al. 1993; Gratton et al. 2010) and thus, it is not surprising that Bellazzini et al. (2012) also found a significant correlation between the $[\mathrm{Fe} / \mathrm{H}]$ abundance and the $A_{\text {rot }} / \sigma_{0}$ ratio. They noted, however, that the correlation with metallicity is not as strong as the dependence on the HB morphology and it is unlikely to be the sole parameter. The Spearman rank correlation coefficient determined by (Bellazzini et al. 2012) for each of the discussed relations is shown in Fig. 13 to give a feeling for their significance. NGC 4372 is one of the most metal-poor GCs in the Galaxy and Fig. 13 shows that it has a somewhat larger amount of ordered motion for its metallicity. That brings it in line with other outliers on this diagram like NGC 7078 (M 15) and NGC 4590.

Age is widely accepted as being the second most important parameter that shapes the HB (e.g. Searle \& Zinn 1978; Lee et al. 1994; Mackey \& Gilmore 2004; Mackey \& van den Bergh 2005 , McDonald \& Zijlstra, in prep.) and is likely to have a significant impact on the rotation of GCs in the sense that older clusters are expected to have dissipated their angular momentum through dynamical relaxation, or could be slowed down by tidal interactions with the Galaxy (Goodwin 1997). In this respect, one could see the left panel of Fig. 13 as an ensemble of slowly rotating old halo GCs with blue HBs and their rapidly rotating counterparts of young halo clusters with extended or red HBs.

Additionally, Bellazzini et al. (2012) suggested a weak inverse relation between $A_{\text {rot }} / \sigma_{0}$ and the inter-quartile range (IQR) of the $[\mathrm{Na} / \mathrm{O}]$ abundance ratio. The latter is a proxy for the extent of the $\mathrm{Na}$ and $\mathrm{O}$ variations and thus is an indicator of the occurrence of multiple populations in GCs (see Carretta et al. 2009; Gratton et al. 2012). While this dependence is not very significant (Spearman rank-0.34), it is likely to be a secondary effect of the HB morphology dependence, since the extended spreads of $\mathrm{Na}$ and $\mathrm{O}$ abundances in GCs are linked to the extent of He abundances in GCs stars (D'Antona et al. 2002). Helium is important for shaping the HB, as He-enriched stars reach hotter temperatures at the HB stage and GCs with pronounced multiple populations have generally more extended HBs. If we assumed that the relation between the extent of the $[\mathrm{Na} / \mathrm{O}]$ abundance ratio in a GC and its amount of internal rotation represent a genuine formation process, we would expect to observe a direct dependence instead of a reversed dependence. Indeed, according to the most popular scenarios of GCs formation (D'Ercole et al. 2008; Decressin et al. 2007; Bekki 2010), GCs form a second, dynamically cold, and rapidly rotating stellar population. If this were the case, we would still see the clusters with more numerous, chemically enhanced population to be faster rotators. NGC 4372 shows the typical of GCs $\mathrm{Na}-\mathrm{O}$ anti-correlation as our chemical abundance analysis shows. The value for the $\mathrm{IQR}[\mathrm{Na} / \mathrm{O}]$ shown in Fig. 13 is estimated from abundance measurements of the same GIRAFFE spectroscopic sample presented in this work. The full abundance analysis will be presented in a series of subsequent articles.

The amplitude of internal rotation in GCs is also connected to their absolute magnitudes and central velocity dispersions (see Bellazzini et al. 2012). Both quantities are tightly linked to the clusters total mass. NGC 4372 is not an exception in this respect.

In the end, we explore the age-metallicity relation of GCs with respect to their rotation properties (Fig. 14). Normalised ages are taken from the work of Marín-Franch et al. (2009) based on the Zinn \& West (1984) metallicity scale. We have divided the GCs from Bellazzini et al. (2012) and NGC 4372 into slow and fast rotators according to their $A_{\text {rot }} / \sigma_{0}$ ratio with respect to the prediction of the isotropic, rotating model shown in Fig. 12. GCs lying significantly above the predictions of the model are considered as fast rotators. The two well known branches of GCs in the age-metallicity relation are often interpreted as clusters born in-situ and accreted on a later stage in the Milky Way halo as indicated in the figure (Marín-Franch et al. 2009; Leaman et al. 2013). Figure 14 shows that the majority of fast-rotating clusters are the ones supposedly born in-situ, while the slow rotators occupy the more metal poor branch associated with the accreted GCs.

\section{Summary}

We presented the first in-depth study of the structure and kinematics of the old, metal-poor GC NGC 4372. We used archival deep $V$ - and $I$-band images from the WFI camera mounted at the MPG/ESO $2.2 \mathrm{~m}$ telescope to obtain a CMD of the cluster. NGC 4372 is known to suffer from a severe differential 


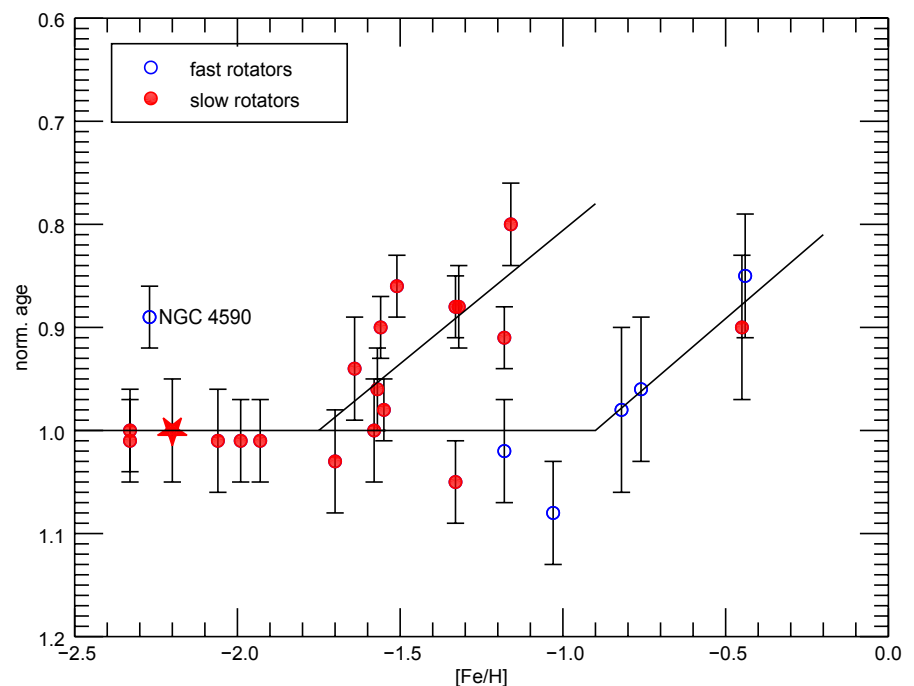

Fig. 14. Age-metallicity relation for GCs with known rotation properties. The normalised ages are taken from Marín-Franch et al. (2009) based on the Zinn \& West (1984) scale. The points are colour-coded based on the $A_{\text {rot }} / \sigma_{0}$ ratio. Slow rotators are marked with red and fast rotators with blue. NGC 4372 is marked with a red star. The two sequences of GCs supposedly accreted into the Milky Way halo and born in-situ are marked with black lines.

reddening. We used a well-established method to correct for it and showed that the mean $E(B-V)$ is 0.5 mag but varies between 0.3 and $0.8 \mathrm{mag}$ across the observed field. We made a cut at $V=20 \mathrm{mag}$ in the extinction corrected CMD and used the resulting catalogue to obtain a number density profile of this GC. Using a MCMC maximum likelihood fitting procedure we derived the centroid of the cluster, its half-light radius $\left(r_{\mathrm{h}}=3.44 \pm 0.04^{\prime}\right)$, ellipticity $(\epsilon=0.08 \pm 0.01)$, and foreground stellar density, by considering a Plummer distribution.

In order to derive the kinematic properties of NGC 4372 , we used high resolution spectroscopic observations from the FLAMES spectrograph at the VLT. Based on precise radial velocity measurements and metallicity estimates, we selected a clean sample of 131 NGC 4372 RGB/AGB stars. With this radial velocity sample we derived a rotation profile extending to the cluster half-light radius. These observations show that the cluster has a significant internal rotation with a maximum amplitude of $A_{\text {rot }}=1.2 \pm 0.25 \mathrm{~km} \mathrm{~s}^{-1}$. On the other hand, the bestfit velocity dispersion profile indicates a central velocity dispersion of $\sigma_{0}=4.56 \pm 0.3 \mathrm{~km} \mathrm{~s}^{-1}$. The resulting $A_{\text {rot }} / \sigma_{0}$ ratio of $0.26 \pm 0.07$ is relatively large for NGC 4372's low metallicity and old age. Our results show that NGC 4372 is flattened in the direction of its internal rotation (i.e. perpendicular to its rotation axis), which is the most likely reason for deviations from sphericity.

Our observational results allow us to construct a realistic dynamical interpretation of NGC 4372. We compared our full set of observations with a family of physically motivated models, based on distribution functions, specifically constructed to describe quasi-relaxed, differentially rotating stellar systems. The best fitting model is a good representation of the number density and velocity dispersion profile of this GC, as well as its differential rotation, however, being highly spherical, the model fails to reproduce the observed flattening of the cluster. Based on this model, the total dynamical mass of NGC 4372 is $\sim 2 \times 10^{5} M_{\odot}$ with a mass-to-light ratio $M / L_{V}$ between 1.4 and $2.3 M_{\odot} / L_{\odot}$. We found that the modelled quantities depend very weakly on the adopted inclination angle.
Finally, we discuss the importance of internal rotation (particularly the $A_{\text {rot }} / \sigma_{0}$ ratio) of NGC 4372 to its morphology and chemical composition by comparing it to similar studies of other GCs (see Bellazzini et al. 2012). We argue that the presumably less relaxed young halo GCs have generally higher $A_{\text {rot }} / \sigma_{0}$ ratios. We also show that NGC 4372 (an archetypical old halo GC) has unusually high $A_{\text {rot }} / \sigma_{0}$ ratio for its low metallicity, but it could still be considered a slow rotator when compared to the young halo GC population. When we consider the two distinct branches of GCs in the age-metallicity relation, we notice that the fast rotating GCs are those presumably born in-situ, while the slow rotators occupy predominantly the branch of the presumably accreted GCs.

Acknowledgements. We thank Benjamin Hendricks for valuable discussions. N.K., A.K., and M.J.F. acknowledge the Deutsche Forschungsgemeinschaft for funding through Emmy-Noether grant Ko 4161/1. C.I.J. gratefully acknowledges support through the Clay Fellowship administered by the Smithsonian Astrophysical Observatory. T.H.P. acknowledges support in the form of a FONDECYT Regular Project Grant (No. 1121005) and from BASAL Center for Astrophysics and Associated Technologies (PFB-06). This work was in part supported by Sonderforschungsbereich SFB 881 "The Milky Way System" (subproject A4) of the German Research Foundation (DFG). This publication makes use of data products from the Two Micron All Sky Survey, which is a joint project of the University of Massachusetts and the Infrared Processing and Analysis Center/California Institute of Technology, funded by the National Aeronautics and Space Administration and the National Science Foundation. This research used the facilities of the Canadian Astronomy Data Centre operated by the National Research Council of Canada with the support of the Canadian Space Agency. This research has made use of NASA's Astrophysics Data System.

\section{References}

Alcaino, G., Liller, W., Alvarado, F., \& Wenderoth, E. 1991, AJ, 102, 159 Baade, D., Meisenheimer, K., Iwert, O., et al. 1999, The Messenger, 95, 15 Baumgardt, H., Grebel, E. K., \& Kroupa, P. 2005, MNRAS, 359, L1 Baumgardt, H., Côté, P., Hilker, M., et al. 2009, MNRAS, 396, 2051 Bekki, K. 2010, ApJ, 724, L99

Bellazzini, M., Bragaglia, A., Carretta, E., et al. 2012, A\&A, 538, A18

Belokurov, V., Evans, N. W., Irwin, M. J., Hewett, P. C., \& Wilkinson, M. I. 2006, ApJ, 637, L29

Bianchini, P., Varri, A. L., Bertin, G., \& Zocchi, A. 2013, ApJ, 772, 67

Binney, J. 2005, MNRAS, 363, 937

Blecha, A., Cayatte, V., North, P., Royer, F., \& Simond, G. 2000, Proc. SPIE, 4008, 467

Boily, C. M. 2000, in Massive Stellar Clusters, eds. A. Lançon, \& C. M. Boily, ASP Conf. Ser., 211, 190

Bradford, J. D., Geha, M., Muñoz, R. R., et al. 2011, ApJ, 743, 167

Cardelli, J. A., Clayton, G. C., \& Mathis, J. 1989, ApJ, 345, 245

Carretta, E., Bragaglia, A., Gratton, R. G., et al. 2009, A\&A, 505, 117

Casetti-Dinescu, D. I., Girard, T. M., Herrera, D., et al. 2007, AJ, 134, 195

Catelan, M. 2009, Ap\&SS, 320, 261

Chen, C. W., \& Chen, W. P. 2010, ApJ, 721, 1790

Cohn, H., \& Hut, P. 1984, ApJ, 277, L45

Cutri, R. M., Skrutskie, M. F., van Dyk, S., et al. 2003, VizieR Online Data Catalog: II/246

D’Antona, F., Caloi, V., Montalbán, J., Ventura, P., \& Gratton, R. 2002, A\&A, 395,69

D’Ercole, A., Vesperini, E., D’Antona, F., McMillan, S. L. W., \& Recchi, S. 2008, MNRAS, 391, 825

Da Costa, G. S. 1982, AJ, 87, 990

Davies, R. L., Efstathiou, G., Fall, S. M., Illingworth, G., \& Schechter, P. L. 1983, ApJ, 266, 41

Davoust, E. 1986, A\&A, 166, 177

Decressin, T., Meynet, G., Charbonnel, C., Prantzos, N., \& Ekström, S. 2007, A\&A, 464, 1029

Dejonghe, H. 1987, MNRAS, 224, 13

Djorgovski, S., \& King, I. R. 1984, ApJ, 277, L49

Emsellem, E., Cappellari, M., Krajnović, D., et al. 2011, MNRAS, 414, 888 Erben, T., Schirmer, M., Dietrich, J. P., et al. 2005, Astron. Nachr., 326, 432 Ernst, A., Glaschke, P., Fiestas, J., Just, A., \& Spurzem, R. 2007, MNRAS, 377, 465

Fiestas, J., Spurzem, R., \& Kim, E. 2006, MNRAS, 373, 677

Fusi Pecci, F., Ferraro, F. R., Bellazzini, M., et al. 1993, AJ, 105, 1145

Geisler, D., Piatti, A. E., Claria, J. J., \& Minniti, D. 1995, AJ, 109, 605 
Gerashchenko, A. N., Kadla, Z. I., \& Malakhova, Y. N. 1999, Astron. Rep., 43, 20

Geyer, E. H., Nelles, B., \& Hopp, U. 1983, A\&A, 125, 359

Gnedin, O. Y., Lee, H. M., \& Ostriker, J. P. 1999, ApJ, 522, 935

Goodwin, S. P. 1997, MNRAS, 286, L39

Gratton, R. G., Carretta, E., Bragaglia, A., Lucatello, S., \& D’Orazi, V. 2010, A\&A, 517, A81

Gratton, R. G., Carretta, E., \& Bragaglia, A. 2012, A\&ARv, 20, 50

Gunn, J. E., \& Griffin, R. F. 1979, AJ, 84, 752

Harris, W. E. 1996, AJ, 112, 1487

Hartwick, F. D. A., \& Hesser, J. E. 1973, ApJ, 186, 1171

Hastings, W. K. 1970, Biometrika, 57, 97

Hendricks, B., Stetson, P. B., VandenBerg, D. A., \& Dall'Ora, M. 2012, AJ, 144, 25

Ibata, R., Nipoti, C., Sollima, A., et al. 2013, MNRAS, 428, 3648

Kim, E., Yoon, I., Lee, H. M., \& Spurzem, R. 2008, MNRAS, 383, 2

King, I. R. 1966, AJ, 71, 64

Koch, A., Odenkirchen, M., Grebel, E. K., \& Caldwell, J. A. R. 2004, Astron. Nachr., 325, 299

Küpper, A. H. W., Kroupa, P., Baumgardt, H., \& Heggie, D. C. 2010, MNRAS, 407, 2241

Lane, R. R., Kiss, L. L., Lewis, G. F., et al. 2009, MNRAS, 400, 917

Lane, R. R., Kiss, L. L., Lewis, G. F., et al. 2010, MNRAS, 406, 2732

Lane, R. R., Kiss, L. L., Lewis, G. F., et al. 2011, A\&A, 530, A31

Leaman, R., VandenBerg, D. A., \& Mendel, J. T. 2013, MNRAS, 436, 122

Lee, Y.-W., Demarque, P., \& Zinn, R. 1990, ApJ, 350, 155

Lee, Y.-W., Demarque, P., \& Zinn, R. 1994, ApJ, 423, 248

Mackey, A. D., \& Gilmore, G. F. 2004, MNRAS, 355, 504

Mackey, A. D., \& van den Bergh, S. 2005, MNRAS, 360, 631

Mackey, A. D., Da Costa, G. S., Ferguson, A. M. N., \& Yong, D. 2013, ApJ, 762, 65

Mandushev, G., Staneva, A., \& Spasova, N. 1991, A\&A, 252, 94

Marín-Franch, A., Aparicio, A., Piotto, G., et al. 2009, ApJ, 694, 1498

Martin, N. F., de Jong, J. T. A., \& Rix, H.-W. 2008, ApJ, 684, 1075

Mashchenko, S., \& Sills, A. 2005a, ApJ, 619, 243

Mashchenko, S., \& Sills, A. 2005b, ApJ, 619, 258

Mathis, J. S. 1990, ARA\&A, 28, 37

McLaughlin, D. E., \& van der Marel, R. P. 2005, ApJS, 161, 304

Milone, A. P., Piotto, G., Bedin, L. R., et al. 2012, A\&A, 537, A77
Momany, Y., Vandame, B., Zaggia, S., et al. 2001, A\&A, 379, 436 Muñoz, R. R., Padmanabhan, N., \& Geha, M. 2012, ApJ, 745, 127 Newell, B., \& Oneil, Jr., E. J. 1978, ApJS, 37, 27

Odenkirchen, M., Grebel, E. K., Rockosi, C. M., et al. 2001, ApJ, 548, L165 Pasquini, L., Avila, G., Blecha, A., et al. 2002, The Messenger, 110, 1

Pietrinferni, A., Cassisi, S., Salaris, M., \& Castelli, F. 2004, ApJ, 612, 168 Pietrinferni, A., Cassisi, S., Salaris, M., \& Castelli, F. 2006, ApJ, 642, 797 Piotto, G., King, I. R., Djorgovski, S. G., et al. 2002, A\&A, 391, 945 Plummer, H. C. 1911, MNRAS, 71, 460

Pryor, C., \& Meylan, G. 1993, in Structure and Dynamics of Globular Clusters, eds. S. G. Djorgovski, \& G. Meylan, ASP Conf. Ser., 50, 357

Robin, A. C., Reylé, C., Derrière, S., \& Picaud, S. 2003, A\&A, 409, 523 Rosenberg, A., Piotto, G., Saviane, I., \& Aparicio, A. 2000, A\&AS, 144, 5 Rutledge, G. A., Hesser, J. E., Stetson, P. B., et al. 1997, PASP, 109, 883

Satoh, C. 1980, PASJ, 32, 41

Schirmer, M. 2013, ApJS, 209, 21

Schlafly, E. F., \& Finkbeiner, D. P. 2011, ApJ, 737, 103

Schlegel, D. J., Finkbeiner, D. P., \& Davis, M. 1998, ApJ, 500, 525 Searle, L., \& Zinn, R. 1978, ApJ, 225, 357

Servillat, M., Webb, N. A., \& Barret, D. 2008, A\&A, 480, 397

Skrutskie, M. F., Cutri, R. M., Stiening, R., et al. 2006, AJ, 131, 1163

Sollima, A., Bellazzini, M., \& Lee, J.-W. 2012, ApJ, 755, 156

Stetson, P. B. 1987, PASP, 99, 191

Stetson, P. B. 1993, in Stellar Photometry - Current Techniques and Future Developments, eds. C. J. Butler, \& I. Elliott, IAU Colloq., 136, 291

Stetson, P. B. 2000, PASP, 112, 925

Stetson, P. B. 2005, PASP, 117, 563

Tonry, J., \& Davis, M. 1979, AJ, 84, 1511

Trager, S. C., King, I. R., \& Djorgovski, S. 1995, AJ, 109, 218

Trenti, M., \& van der Marel, R. 2013, MNRAS, 435, 3272

van de Ven, G., van den Bosch, R. C. E., Verolme, E. K., \& de Zeeuw, P. T. 2006, A\&A, 445, 513

van den Bergh, S. 2008, AJ, 135, 1731

Varri, A. L., \& Bertin, G. 2012, A\&A, 540, A94

Walker, M. G., Mateo, M., Olszewski, E. W., et al. 2006, AJ, 131, 2114

White, R. E., \& Shawl, S. J. 1987, ApJ, 317, 246

Wilson, C. P. 1975, AJ, 80, 175

Zinn, R., \& West, M. J. 1984, ApJS, 55, 45

Zocchi, A., Bertin, G., \& Varri, A. L. 2012, A\&A, 539, A65 